\title{
Arthroscopic repair of the subscapularis tendon: indications, limits and technical features
}

\author{
Leonardo Osti ${ }^{1}$ \\ Francesco Soldati \\ Angelo Del Buono1 \\ Matteo Buda² \\ 1 Unit of Arthroscopic and Sports Medicine, Hesperia \\ Hospital, Modena, Italy \\ 2 Unit of Orthopaedics and Traumatology, University \\ of Ferrara, Italy
}

\author{
Corresponding author: \\ Leonardo Osti \\ Unit of Arthroscopy and Sports Medicine \\ Hesperia Hospital \\ Via Arquà, $80 / a$ \\ 41125 Modena, Italy \\ E-mail: leonardoosti@yahoo.com
}

\section{Summary}

The rationale to anatomically repair this tendon is to restore the functional biomechanics of the shoulder. Clinical and imaging assessment are required before undertaking arthroscopy. In this way, associated pathologies of the biceps and labrum may be successfully addressed. The arthroscopic repair of the tendon implies to use suture anchors and reinsert the tendon itself over the footprint. Results after arthroscopy are comparable to those observed after open procedures.

KEY WORDS: subscapularis, injury, arthroscopy, repair.

\section{Introduction}

Subscapularis tendon tears have been firstly described by Smith ${ }^{1}$ and Codman ${ }^{2}$. Hauser reported in 1954 the first case of surgical repair of the subscapularis tendon ${ }^{3}$. Isolated and associated subscapularis lesions are present in 2 to $10 \%$ of all the population, up to $20 \%{ }^{4}$. Many surgical approaches may be use, but the standard repair which better restore its anatomy is arthroscopic ${ }^{5,6}$.

\section{Pathogenesis}

The etiology of sub-scapularis tendon tears is traumatic or degenerative. Traumatic tears are usually secondary to a forced external rotation or extension of the shoulder with the arm abducted. These tears are more frequent in young patients, rarely in elderly, as a consequence of a shoulder dislocation ${ }^{7,8}$. Adolescents are less frequently affected. In degenerative lesions, an associated sub-coracoid impingement may injury the anterosuperior portion of the rotator cuff, and involve the subscapularis tendon. When the coraco-humeral distance is reduced, observed on MRI or CT scans, other structures such as the long head of the biceps tendon and all the rotator cuff tendons may be differently involved. When this distance, which normally ranges from $8.7 \mathrm{~mm}$ to $11 \mathrm{~mm}$, is lower than $5 \mathrm{~mm}$, the risk that the subscapularis tendon is torn is high ${ }^{9,10}$.

The coracoid impingement may be primary or acquired. It is primary, or idiopathic, when associated with congenital abnormalities of the coracoids such as the presence of a lateralized coracoid process ${ }^{11}$, calcifications or ossifications of the subscapularis tendon, subscapularis muscle hypertrophy, and ganglion cysts $^{12}$. It may be secondary to displaced humeral or scapular fractures, non unions, posterior dislocation of the sterno-clavicular joint or iatrogenic, after surgery. Specifically, the Bristow/Latarjet and Trillat procedures change the biomechanics between coracoid and lesser tuberosity ${ }^{13}$; the posterior glenoid osteotomy may increase the anteversion of the glenoid neck and the coraco-glenoid angle, decreasing the coracohumeral distance ${ }^{14}$. The subcoracoid stenosis may also be consequence of a condition of minor anterior instability of the shoulder ${ }^{15}$, rotator cuff insufficiency, and sick scapula syndrome ${ }^{16}$. When an anterior acromioplasty is undertaken in patients with rotator cuff disfunction and narrow coraco-humeral distance, the antero-superior migration of the humeral head may predispose to the subcoracoid impingement syndrome ${ }^{17}$.

Finally, given the anatomical contiguity of the medial margin of the pulley and the subscapularis tendon insertion site, the subscapularis is torn in $63 \%$ of patients in whom the LHB is sub-luxated or dislocated ${ }^{9}$.

\section{Tear classification}

The subscapularis tendon tears may be classified as partial and complete, retracted and not retracted, superior (involving the upper third) and inferior (extended to the lower third 4-5). The classification system by Lafosse tendon distinguishes 5 types of insertional tears $^{18}$. A type I tear is a simple erosion of the upper third of the tendon without any disconnection to the 
bone; a type II is a frank detachment of the upper portion of the tendon; a type III lesion is characterized by involvement of all the insertion of the tendon without detachment of the lower third of the muscular portion. In type IV tears, the subscapularis tendon is completely detached from the lesser tuberosity and the humeral head is centered within the joint. In a type $\mathrm{V}$ lesion, the lesion is complete, the humeral head is translated anteriorly and superiorly, with coracoid impingement and fatty degeneration of the muscle fibers of the subscapularis.

\section{Clinical assessment}

On clinical assessment, the shoulder pain related to a subscapularis tendon tear is more anterior compared to the typical pain observed in patients with rotator cuff tears. Weakness, mostly in internal rotation, and impaired function to touch the lumbar spine with the hand may also be present. At times, is severe lesion, the shoulder may be dislocated or sub-luxated anteriorly ${ }^{19}$. Lift-off, belly-press, Napoleon and bear-hug are specific tests to assess the subscapularis tendon ${ }^{28}$. Barth et al. ${ }^{20}$ have reported that the bear-hug test has the highest sensitivity $(60 \%)$, followed by the belly-press $(40 \%)$, Napoleon (25\%); and lift-off test (17.6\%). On the contrary, all the tests are highly specific (lift-off test 100\%; Napoleon test $97.3 \%$; belly-press test $97.3 \%$; bear-hug test $91.7 \%$ ). All these tests allow to diagnose a partial tear in $30 \%$ of cases. More than $50 \%$ of tendon thickness is torn when the Napoleon test is positive; more than $75 \%$ when the lift-off test is positive.

The examiner should assess any internal rotation deficiency (less than $90^{\circ}$ ) related to an excessive tension of the posterior -superior aspect of the capsule. In such instances, all tests may be positive (false-positive) as the a condition of limited internal rotation may mimic a loss of strength of the subscapularis tendon.

The lift-off test described by Gerber ${ }^{21}$ is performed placing the hand of the affected side behind the back, at the level of the lumbar spine, asking the patient to rotate the arm internally and to lift the hand from the back, posteriorly. It is positive when the patient is not able to raise the hand or if this movement is positive only with the elbow extended. This test has limited value in clinical practice as many conditions may not allow to reach this position because of pain and limited range of motion of the shoulder.

The belly-press test ${ }^{22}$ starts with the arm beside the body and the elbow flexed to $90^{\circ}$. The patient is asked to press the hand against the belly with the shoulder rotated internally. The test is considered positive $(A)$ if the pressing force is weaker than contra-laterally (tested by pushing on his elbows), or (B) if the pressing movement of the hand against the belly is possible only with the elbow extension and shoulder extended. This indicates a deficiency of the scapularis tendon to rotate internally the shoulder, and produce a pushing force against the abdomen.

The Napoleon test is a modified belly-press test, with the hand lying on the abdomen, in the same position which Napoleon Bonaparte used to be portrayed. The test is negative, or normal, if the patient is able to press his hand against the belly with the wrist in straight position; it is positive when the patient is able to push the belly with the wrist flexed to $90^{\circ}$, or $30^{\circ}$ to $60^{\circ}$.

The bear-hug test ${ }^{23}$ is performed with the palm of the hand of the affected side on the opposite shoulder, the fingers extended, and the elbow ahead of the body with the shoulder slightly flexed. The patient is asked to keep the position (forced internal rotation) while the examiner attempts to pull the hand of the patient away from the opposite shoulder by applying a force in external rotation perpendicular to the forearm. The test is positive when the patient cannot keep the hand on the opposite shoulder or the strength in internal rotation is impaired compared to the opposite side. The test is negative when the strength is comparable to the contra-lateral side, with no pain. This diagnosis is supported when the external rotation is pathologically increased ${ }^{23}$.

\section{Imaging}

Ultrasound (US) is less reliable than MRI. US is preferred to assess the tendon repaired after shoulder arthroscopy. Arthro-MRI provides higher diagnostic reliability compared to conventional MRI in patients with subscapularis tendon tears, with 40 to $100 \%$ sensitivity. An indirect sign, often associated with partial subscapularis tears, is a medial dislocation of the long head of the biceps.

Fatty degeneration (fatty infiltration) is negative prognostic factor for full functional recovery of the shoulder. The percentage of fatty infiltration predictive of success after cuff repair is lower than $75 \%$.

Tung et al. ${ }^{24}$ have reported that only $31 \%$ of subscapularis tears confirmed at arthroscopy had been preoperatively on MRI. On the other hand, arthro-MRI can be better assess both supraspinatus and infraspinosus tears, accounting for $71 \%$ to $100 \%$ sensitivity ${ }^{25}$. Given the poor sensitivity of conventional MRI (36\%) to diagnose subscapularis tendon tears, and the higher sensitivity after contrast injection, MRI and arthro-MRI have been compared in patients with tears involving more than $30 \%$ of the subscapularis tendon thickness ${ }^{26}$. By considering arthroscopy as standard assessment of these lesion, it has been shown low increase of sensitivity of arthro-MRI (40 vs $36 \%)^{26}$.

\section{Indications to arthroscopic repair}

Burkhart and Tehrany reported the first series of patients undergoing arthroscopic repair of subcsapularis tendon. Repair is important to restore not only the internal rotation of the shoulder but also a dynamic anterior stabilization of the joint provided by a fine balance of pairs of forces. In addition, other studies highlighted that the integrity of the subscapularis tendon plays a key role to reduce recurrences of rotator 
cuff tears 27,28 . Nevertheless, good results may be found after simple arthroscopic debridement in patients with isolated irreparable tears.

\section{Arthroscopic repair procedure}

Arthroscopic subscapularis repair can be performed with the patient in lateral or beach-chair position. Arthroscopy allows a complete visualization of intraarticular aspects of the joint. Other features are also recommended: 1) The so-called "posterior lever push": the assistant applies a lever from anterior to posterior to better visualize the tendon and the extent of the lesion. 2) The internal rotation of the arm may better expose the tendon tear. 3) The use of an additional $70^{\circ}$ arthroscope may also improve the joint view. Paribelli19 also introduced an approach to the joint through the sub-acromial space to visualize and assess the axillary nerve.

The approach to the subscapularis tendon is mandatory to carefully assess the long head of the biceps. Frequently involved in patients with subscapularis tendon tears, the LHB may be unstable at the pulley, completely or partially degenerated, and torn.

A LHB tenotomy is indicated in most patients when necessary. A functional tenodesis at the groove may be undergone in young active patients, before or after the subscapularis tendon repair. We prefer to perform first the tenodesis at the biceps groove. Rotator cuff or labral tears following a trauma should be addressed concomitantly.

Coracoplasty and release/opening of the rotator interval may be performed. It is indicated in patients with coracoid impingement and related severe restriction of the subcoracoid space. This procedure is expected to induce a great release of local growth factors, as observed after acromioplasty. It is necessary to make an anterior-superior working portal by evaluating the optimal direction of the suture anchor position using a spinal needle pointing the footprint of the subscapularis tendon ("deadman angle" / 45 inclination).

Release of the adhesions is also important, especially when the lesions are retracted. It can be anterior, between the coracoids and the subscapularis tendon, or posterior, between the glenoid and the subscapularis tendon. However, undertaking a medial release through surrounding neuro-vascular structures may be controversial but, when performed, radiofrequencies should be done. Once the subscapularis tear has been identified, the retracted tendon can be loaded with a traction wire that may temporary favor the reduction of the tendon over the footprint, and assess accurately the tendon elasticity to repair it with a physiological tension.

Then, the footprint is prepared using the same principles adopted to repair the rotator cuff. The bone surface is cautiously decorticated and the footprint and subchondral bone exposure should be "medialized" up to $7 \mathrm{~mm}$ when lesion are retracted. At this stage, microfractures may improve the biological response at the bone-tendon interface ${ }^{29}$.
Anchors (absorbable or non-absorbable) have to be placed and used in the same fashion of those implanted for rotator cuff repair. Biomechanically, it is advisable to use an anchor for each square centimeter of the bare footprint area: a single anchor may be usually used to complete the repair in almost all instances. As already reported in rotator cuff surgery, a double row repair is proven to be biomechanically advantageous in terms of strength and load to failure. Knotless anchors or bridging sutures can be alternatively used. The same tools used to repair the rotator cuff may be used to pass the sutures, paying attention that the sub-coracoid space is far narrower than the sub-acromial space. Double layer and splitting tears need to be addressed using small instruments which pass within the tendon itself without increasing the lesion neither compromising the primary stability of the repair. In partial tears, instrumental and arthroscopic diagnosis improve sensitivity and specificity of diagnosis, allowing to undertake a transtendineous repair similarly to what is made in "PASTA" lesions of the rotator cuff 28 (Figs. 1-6).

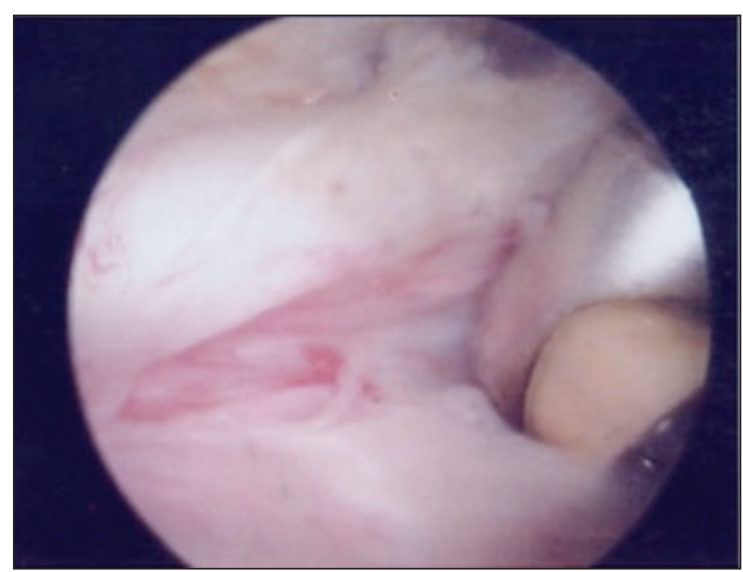

Figure 1. Lesion of the subscapularis tendon: releasing the adhesions.

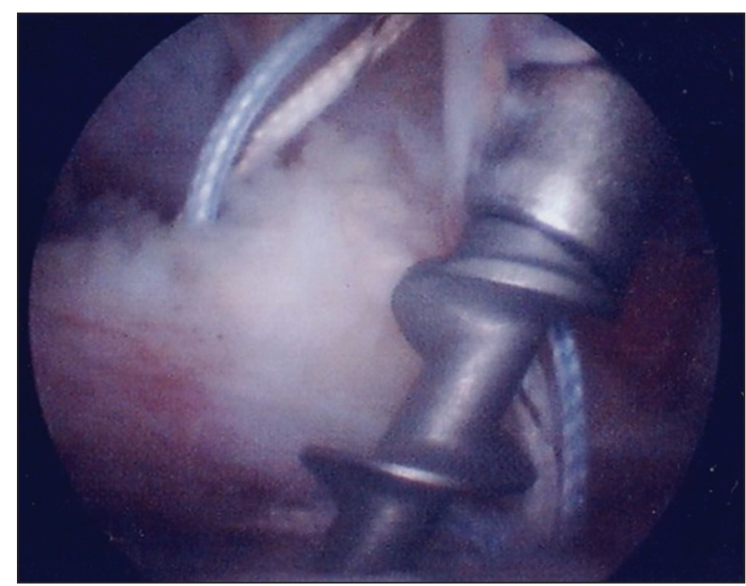

Figure 2. Inserting the anchor in a double anchor subscapularis tendon repair. 


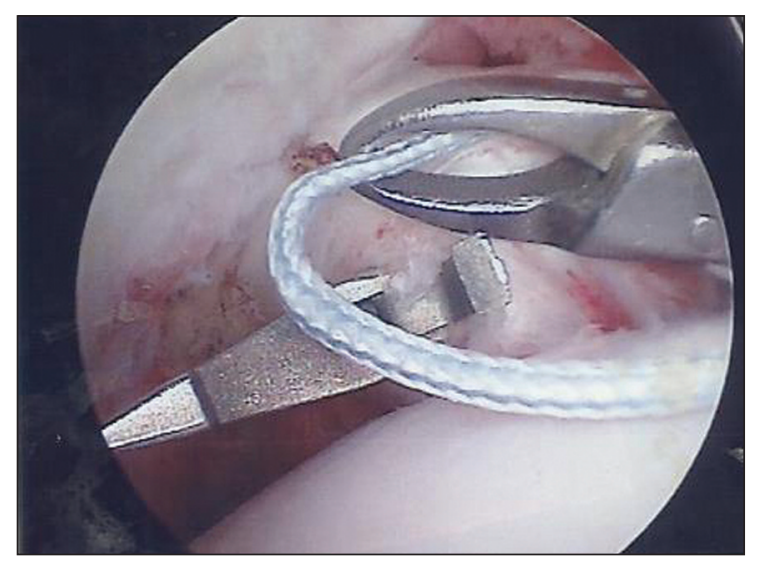

Figure 3. Passing the suture with a tissue penetrator.

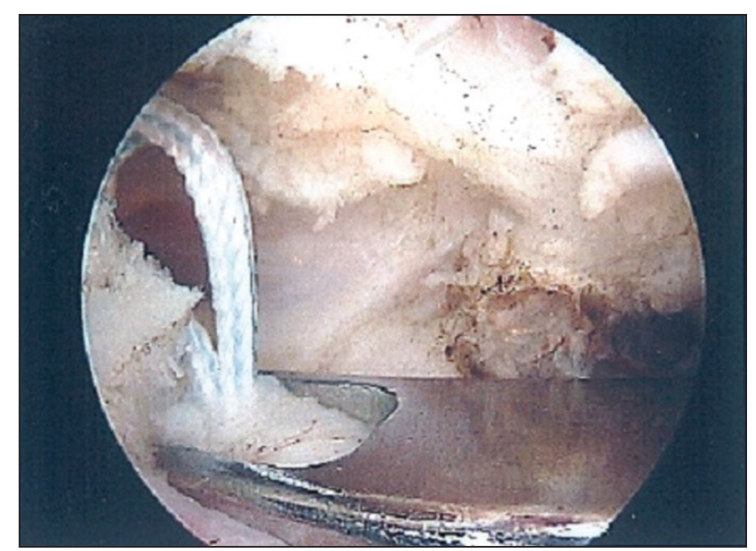

Figure 4. Passing the suture with a suture needle pounch.

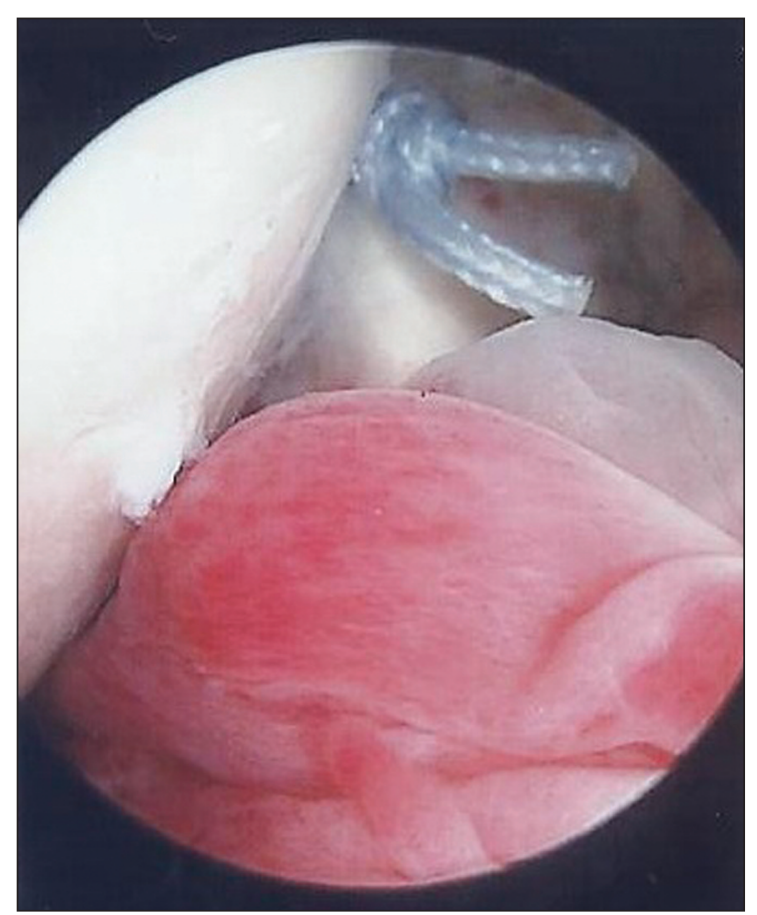

Figure 5. Arthroscopic intrarticular view of repaired subscapularis tendon.

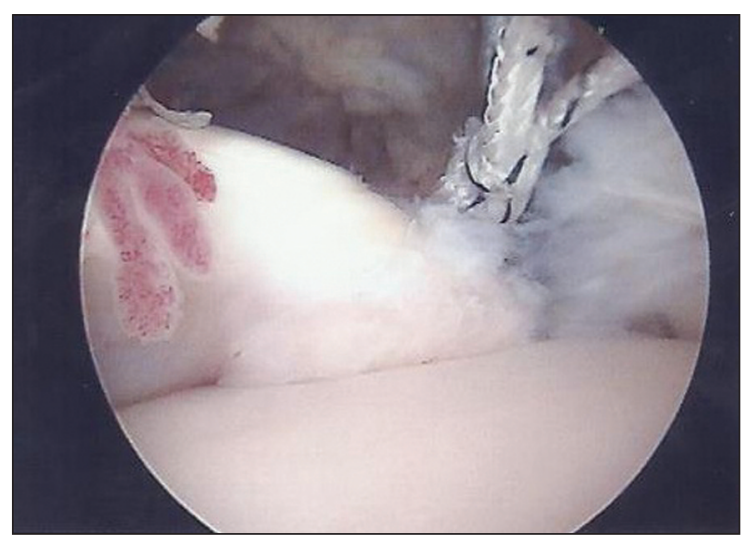

Figure 6. Complete coverage of the footprint in a repaired subscapularis tendon.

\section{Results}

Results of isolated arthroscopic repair reported in peer reviewed Literature are summarized in Table 1.

\section{Limits of arthroscopic repair}

Limitation to repair arthroscopically a tear are the extent or grade of the lesion, the tissue retraction and the quality of the tendon itself. In some instances, the procedure has to be converted into an open procedure to reduce the extent of tissue infiltration, frequent in arthroscopy ${ }^{27}$. The main limit to repair the subscapularis tendon is the extension of the lesion ${ }^{30}$ Open surgery is indicated for management of extraarticular lesions or tears involving the myotendineus portion. In these cases, an anatomical repair is not mandatory as it provides a tenodesis effect, eventually achieved using augmentation with autologous or eterologous tendon grafts, or with muscle-tendon transfers (i.e. the pectoralis maior).

Different tissue engineered biomaterials have been introduced for augmentation, using a combination of principles of engineering and biology: artificial polymers, biodegradable films, and biomaterials derived from animals or human. Synthetic scaffolds come from chemical compounds to better control both chemical and physical properties and confer relatively stronger mechanical strength and consistency.

\section{Postoperative care and rehabilitation}

Rehabilitation of subscapularis tears follows the same principles of rotator cuff rehabilitation. Specifically, brace for 3 to 6 weeks, early or delayed (after 3-4 weeks) passive assisted motion; inhibition of external rotation for 6 weeks, strenghtening activities after 3 months and return to manual work and sports after at least 4-6 months.

In general, the rehabilitation program should carefully consider the extent of the tissue retraction and the tendon condition in terms of resistance and elasticity. 


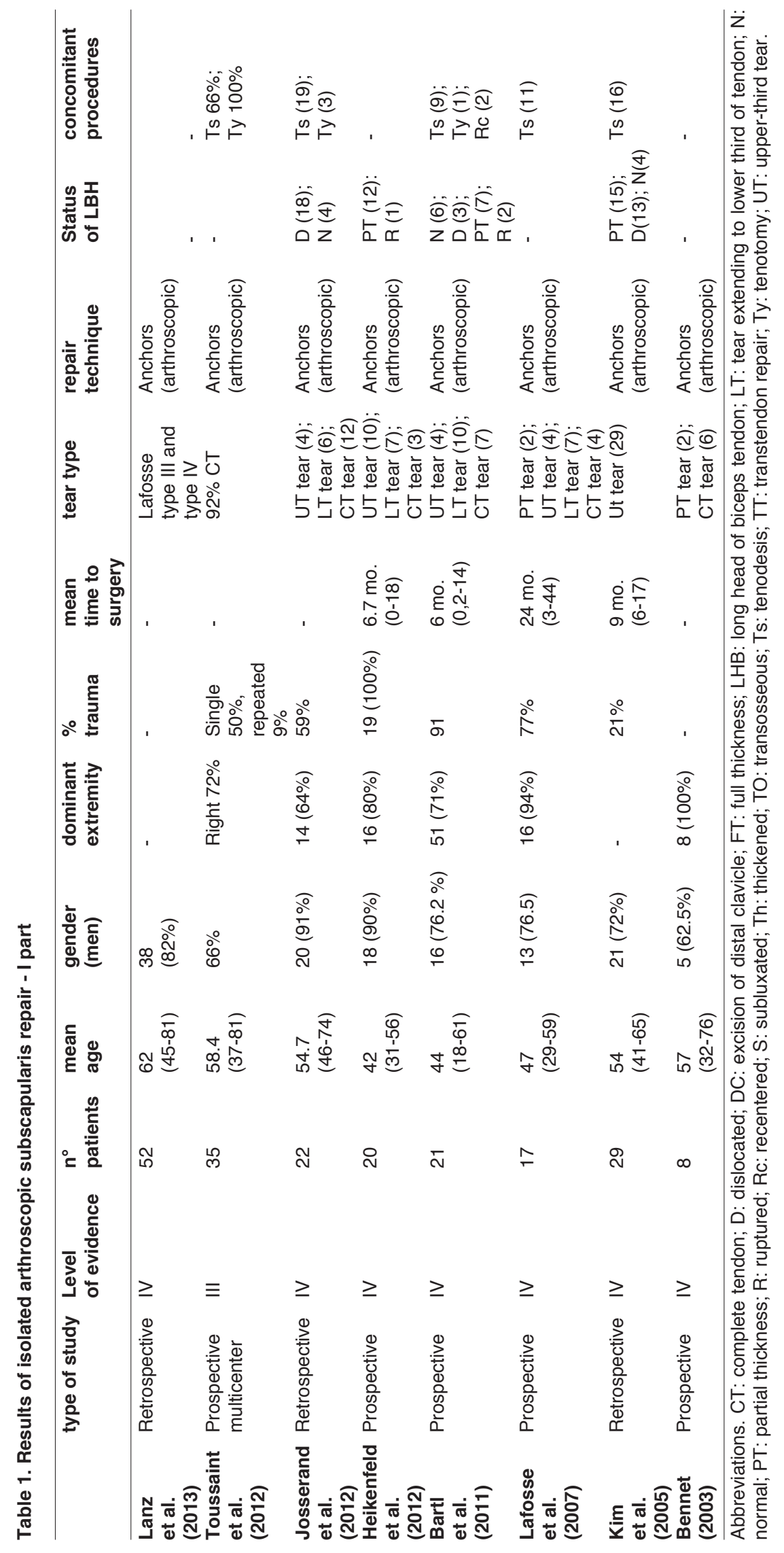




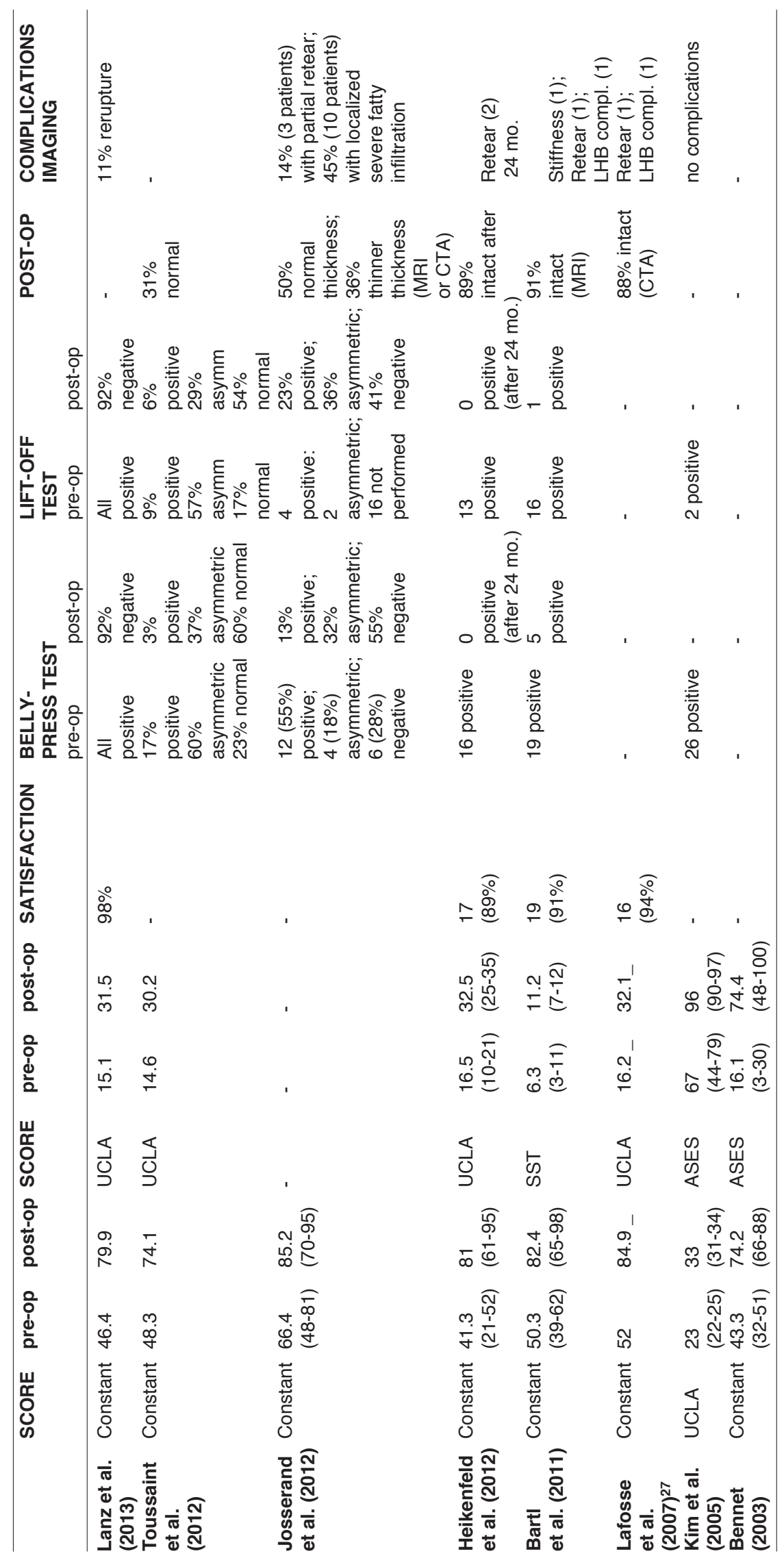




\section{References}

1. Smith J. Pathological appearances of seven cases of injury of the shoulder joint, with remarks. London Med Gazette 1834; 14:280.

2. Codman E. Complete rupture of the supraspinatus tendon: Operative treatment with report of two successful cases. Boston Med Surg 20:708-710.

3. Hauser E. Avulsion of the tendon of the subscapularis muscle. J Bone Joint Surg Am 1954; 36: 139-141.

4. Arai R, Sugaya H, Mochizuki T, Nimura A, Moriishi J, Akita K. Subscapularis tendon tear: an anatomic and clinical investigation. Arthroscopy 2008; 24:997-1004.

5. Flury M, John M, Goldhahn J, Schwyzer H, Simmen B. Rupture of the subscapularis tendon (isolated or in combination with supraspinatus tear): when is a repair indicated? J Shoulder Elbow Surg 2006; 15:659-664.

6. Bartl C, Scheibel M, Magosch P, Lichtenberg S, Habermeyer P. Open repair of isolated traumatic subscapularis tendon tears. Am J Sports Med 2011; 39:490-496.

7. Kato S FH, Kan I, Yoshida M, Kasama K, Marumo K. Incomplete joint side tear of the subscapularis tendon with a small fragment in an adolescent tennis player: a case report. Sports Med Arthrosc Rehabil Ther Technol 2012; 4:24.

8. Bhalla A, Higashigawa K, McAllister D. Subscapularis tendon rupture in an 8-year-old boy: a case report. Am J Orthop (Belle Mead NJ) 2011; 40:471-474.

9. Denard P, Lädermann A, Burkhart S. Arthroscopic management of subscapularis tears. Sports Med Arthrosc 2011; 19:333-341.

10. Foad $A$, Wijdicks $C$. The accuracy of magnetic resonance imaging and magnetic resonance arthrogram versus arthroscopy in the diagnosis of subscapularis tendon injury. Arthroscopy 2012; 28:636-641.

11. Gumina S, Postacchini F, Orsina L, Cinotti G. The morphometry of the coracoid process - its aetiologic role in subcoracoid impingement syndrome. Int Orthop 1999; 23:198201.

12. Martetschläger F, Rios D, Boykin R, Giphart J, de Waha A, Millett P. Coracoid impingement: current concepts. Knee Surg Sports Traumatol Arthrosc 2012; 20:2148-2155.

13. Dines D, Warren R, Inglis A, Pavlov H. The coracoid impingement syndrome. J Bone Joint Surg Br 1990; 72:314316.

14. Freehill M. Coracoid impingement: diagnosis and treatment. J Am Acad Orthop Surg 2011; 19: 191-197.

15. Garofalo R, Conti M, Massazza G, Cesari E, Vinci E, Castagna A. Subcoracoid impingement syndrome: a painful shoulder condition related to different pathologic factors. Mus- culoskelet Surg 2011; 95(Suppl 1):S25-29.

16. Kibler W, Dome D. Internal impingement: concurrent superior labral and rotator cuff injuries. Sports Med Arthrosc 2012; 20:30-33.

17. Suenaga N, Minami A, Kaneda K. Postoperative subcoracoid impingement syndrome in patients with rotator cuff tear. J Shoulder Elbow Surg 2000 Jul-Aug; 9(4):275-278.

18. Lafosse L, Jost B, Reiland Y, Audebert S, Toussaint B, Gobezie R. Structural integrity and clinical outcomes after arthroscopic repair of isolated subscapularis tears. J Bone Joint Surg Am 2007; 89:1184-1193.

19. Paribelli G, Boschi S. The injuries of the subscapularis. G. Di Giacomo The rotator cuff 2010; G. Di Giacomo The rotator cuff.

20. Barth J, Audebert S, Toussaint B, Charousset C, Godeneche A, Graveleau N, Joudet T, Lefebvre Y, NoveJosserand L, Petroff E, Solignac N, Scymanski C, Pitermann M, Thelu C. Society FA. Diagnosis of subscapularis tendon tears: are available diagnostic tests pertinent for a positive diagnosis? Orthop Traumatol Surg Res 2012; 98:S178-185.

21. Gerber C, Krushell R. Isolated rupture of the tendon of the subscapularis muscle. Clinical features in 16 cases. J Bone Joint Surg Br 1991; 73:389-394.

22. Gerber $\mathrm{C}$, Hersche $\mathrm{O}$, Farron $\mathrm{A}$. Isolated rupture of the subscapularis tendon. J Bone Joint Surg Am 1996; 78:1015-1023.

23. Barth J, Burkhart S, De Beer J. The bear-hug test: a new and sensitive test for diagnosing a subscapularis tear. Arthroscopy 2006; 22:1076-1084.

24. Tung G, Yoo D, Levine S, Brody J, Green A. Subscapularis tendon tear: primary and associated signs on MRI. J Comput Assist Tomogr 2001; 25:417-424.

25. Pfirrmann C, Zanetti M, Weishaupt D, Gerber C, Hodler J. Subscapularis tendon tears: detection and grading at MR arthrography. Radiology 1999; 213:709-714.

26. Adams C, Schoolfield J, Burkhart S. Accuracy of preoperative magnetic resonance imaging in predicting a subscapularis tendon tear based on arthroscopy. Arthroscopy 2010; 26:1426-1433.

27. Osti L, Del Buono A, Maffulli N. Microfractures at the rotator cuff footprint: a randomised controlled study. Int Orthop 2013 Jun 13.

28. Osti L, Rizzello G, Panascì M, Denaro V, Maffulli N. Full thickness tears: retaining the cuff. Sports Med Arthrosc 2011; 19:409-419.

29. Lafosse L, Lanz U, Saintmard B, Campens C. Arthroscopic repair of subscapularis tear: Surgical technique and results. Orthop Traumatol Surg Res 2010; 96:S99-108.

30. Ticker J, Burkhart S. Why repair the subscapularis? A logical rationale. Arthroscopy 2011; 27: 1123-1128. 